(2) Open Access Full Text Article

\title{
A novel local anesthetic system: transcriptional transactivator peptide-decorated nanocarriers for skin delivery of ropivacaine
}

This article was published in the following Dove Press journal:

Drug Design, Development and Therapy

28 June 2017

Number of times this article has been viewed

\author{
Chuanyu Chen \\ Peijun You \\ Department of Anesthesiology, \\ Shandong Jining No I People's \\ Hospital, Jining, Shandong, \\ People's Republic of China
}

Correspondence: Peijun You Department of Anesthesiology, Shandong Jining No I People's Hospital, 6 Jiankang Road, Jining 2720II, Shandong, People's Republic of China Email youpjjnph@I63.com
Purpose: Barrier properties of the skin and physicochemical properties of drugs are the main factors for the delivery of local anesthetic molecules. The present work evaluates the anesthetic efficacy of drug-loaded nanocarrier (NC) systems for the delivery of local anesthetic drug, ropivacaine (RVC).

Methods: In this study, transcriptional transactivator peptide (TAT)-decorated RVC-loaded NCs (TAT-RVC/NCs) were successfully fabricated. Physicochemical properties of NCs were determined in terms of particle size, zeta potential, drug encapsulation efficiency, drug-loading capacity, stability, and in vitro drug release. The skin permeation of NCs was examined using a Franz diffusion cell mounted with depilated mouse skin in vitro, and in vivo anesthetic effect was evaluated in mice.

Results: The results showed that TAT-RVC/NCs have a mean diameter of $133.2 \mathrm{~nm}$ and high drug-loading capacity of $81.7 \%$. From the in vitro skin permeation results, it was observed that transdermal flux of TAT-RVC/NCs was higher than that of RVC-loaded NCs (RVC/NCs) and RVC injection. The evaluation of in vivo anesthetic effect illustrated that TAT-RVC/NCs can enhance the transdermal delivery of RVC by reducing the pain threshold in mice.

Conclusion: These results indicate that TAT-decorated NCs systems are useful for overcoming the barrier function of the skin, decreasing the dosage of RVC and enhancing the anesthetic effect. Therefore, TAT-decorated NCs can be used as an effective transdermal delivery system for local anesthesia.

Keywords: local anesthetic system, ropivacaine, transcriptional transactivator peptide, nanocarriers, skin delivery

\section{Introduction}

Ropivacaine (RVC), a congener of mepivacaine and bupivacaine, belongs to the class of long-acting amide-type local anesthetics (LAs). ${ }^{1}$ Based on eight clinical studies, RVC hydrochloride injection (Naropin ${ }^{\circledR}$; Fresenius Kabi, Lake Zurich, IL, USA) has been approved by the US Food and Drug Administration (FDA) since 1996, indicating for the production of local or regional anesthesia for surgery and for acute pain management. ${ }^{2,3}$ Compared with bupivacaine and other agents in the class, RVC has the following advantages of clinical efficacy and safety: better sensory-motor differential blockade, longer duration, less central nervous system (CNS), and cardiovascular toxicities. ${ }^{4-7}$ Whereas, various clinical studies indicated that RVC was rapidly cleared from the injection site and showed short efficacy of the severity of pain (approximately $2 \mathrm{~h}$ postoperatively). ${ }^{8}$ Moreover, intravenous administration brings poor patient convenience and other toxicities such as arrhythmia. ${ }^{9,10}$ These clinical results have prompted that it was critical to the development BY
hereby accept the Terms. Non-commercial uses of the work are permitted without any further permission from Dove Medical Press Limited, provided the work is properly attributed. For permission for commercial use of this work, please see paragraphs 4.2 and 5 of our Terms (https://www.dovepress.com/terms.php). 
of appropriate delivery systems and different administration routes that circumvent the aforementioned problem.

Drug delivery of LAs via the skin brings a lot of benefits such as delivering the local drug continuously and reducing systemic adverse reactions. ${ }^{11,12}$ Polymeric nanoparticles have been used for skin delivery. ${ }^{13}$ In one study, the effect of the inclusion of flufenamic acid in poly(lactide-co-glycolide) nanoparticles on the transport of flufenamic acid into excised human skin was investigated. ${ }^{14}$ In another study, lidocaineloaded poly(epsilon-caprolactone)-polyethylene glycolpoly(epsilon-caprolactone) (PCL-PEG-PCL) nanoparticles were prepared, and a transdermal lidocaine formulation, lidocaine-loaded PCL-PEG-PCL nanoparticles in F127 hydrogel (Nano-Lido Gel), was demonstrated. ${ }^{15}$ Shim et al ${ }^{16}$ evaluated the effect of hydrodynamic size of self-assembled nanoparticles on skin penetration of minoxidil in vitro and in vivo. Accordingly, polymeric materials had been selected to prepare nanocarriers (NCs) in this study.

Various studies show that transcriptional transactivator peptide (TAT) can successfully enhance the transepidermal absorption of therapeutic molecules for the local therapy. ${ }^{17-19}$ Chen et $\mathrm{al}^{19}$ reported that superoxide dismutase mediated with HIV-TAT could penetrate through stratum corneum and effectively attenuate ultraviolet B (UVB)-induced damages in human skin. Furthermore, Wang et $\mathrm{al}^{20}$ reported that TAT-conjugated and octadecyl-quaternized lysine-modified chitosan polymeric liposomes effectively enhanced skin permeation, thus achieving rapid and effective local transdermal anesthetics. More recently, Wang et $\mathrm{al}^{21}$ used TAT-modified NCs to enhance transepidermal delivery of lidocaine on the mice model and found that the system was useful for overcoming the skin barrier and delivering anesthetic through the skin.

In this study, TAT-conjugated poly(lactide-co-glycolide)polyethylene glycol (TAT-PEG-PLGA) was synthesized. RVC was encapsulated into TAT-PEG-PLGA contained NCs (TAT-decorated, RVC-loaded NCs [TAT-RVC/NCs]), and this polymeric nanoparticle system was used to enhance the local anesthesia of RVC. TAT-RVC/NCs were fabricated and characterized for their size, loading efficiency, stability, and drug release. These NCs were subsequently assessed for in vitro skin permeation and/or in vivo anesthetic/analgesic effects in animal models.

\section{Materials and methods \\ Materials}

TAT peptide with terminal cysteine (Cys-AYGRKKRR QRRR) was obtained from Chinese Peptide Co., Ltd. (Hangzhou, China). Poly(lactide-co-glycolide)-polyethylene glycol-maleimide (PLGA-PEG-Mal) was purchased from Xi'an Ruixi Biological Technology Co., Ltd (Xi'an, China). RVC hydrochloride monohydrate $\geq 98 \%$ (high-performance liquid chromatography [HPLC]) was purchased from Sigma-Aldrich Co. (St Louis, MO, USA). Carbopol 940 (CP; molecular weight [MW] 1,000,000) was provided by Shanghai Renmin Pharmaceutical Factory (Shanghai, China). RVC hydrochloride injection (Naropin) was provided by AstraZeneca plc (London, UK). All other chemicals and reagents were of analytical grade or HPLC grade.

\section{Animals}

Sprague Dawley (SD) rats (350-400 g, 10-12 weeks old) were purchased from the Medical Animal Test Center of Shandong University (Jinan, China) and used for experiments after 1 week of acclimatization. Rats were fed with standard diet, allowed water ad libitum, and housed in an air-conditioned room $\left(22^{\circ} \mathrm{C}-24^{\circ} \mathrm{C}, 12 \mathrm{~h}\right.$ light cycle $)$. Animal experiments were conducted according to the Guide for the Care and Use of Laboratory Animals established by Shandong University. Ethics approval was received from the Medical Ethics Committee of Shandong Jining No 1 People's Hospital (reference number: SDJNPH20161118002).

\section{Synthesis of TAT-PEG-PLGA}

TAT-PEG-PLGA was synthesized using the method described by Koren et al. ${ }^{22}$ Briefly, 300 mg PLGA-PEG-Mal, $100 \mathrm{mg}$ Cys-TAT, and $1 \mathrm{~mL}$ triethylamine were reacted in $20 \mathrm{~mL}$ chloroform at room temperature with gentle stirring for $24 \mathrm{~h}$ at room temperature. The solvent was then dialyzed against demonized water using a membrane with MW cutoff (MWCO) of $13 \mathrm{kDa}$ for $24 \mathrm{~h}$. The product was then lyophilized for use. The reaction progress was monitored by thin-layer chromatography (TLC). ${ }^{23}$ TLC confirmed the formation of TAT-PEG-PLGA as the spot of PLGA-PEG-Mal disappeared after the reaction.

\section{Pretreatment of stabilizer solution}

$\mathrm{CP}$ was first dispersed in distilled water overnight to swell it. Then, it was neutralized by adding the required amount of $1 \mathrm{~mol} / \mathrm{L}$ of $\mathrm{NaOH}$ to adjust the $\mathrm{pH}$ to $7 .{ }^{24}$ Afterward, the $\mathrm{CP}$ solution was diluted with distilled water into a concentration of $0.02 \%(\mathrm{w} / \mathrm{v})$.

\section{Preparation of TAT-RVC/NCs}

Prior to the preparation of NCs, RVC hydrochloride monohydrate was stirred twice with the molar amount of triethylamine in dimethyl sulphoxide (DMSO) for $12 \mathrm{~h}$ to 
obtain lipophilic RVC base. TAT-RVC/NCs (Figure 1) were prepared by a solvent displacement technique. ${ }^{25}$ Briefly, $200 \mathrm{mg}$ of TAT-PEG-PLGA and $50 \mathrm{mg}$ of RVC were accurately weighed and dissolved in $5 \mathrm{~mL}$ acetone. The organic phase was added dropwise into a desired aqueous stabilizer of $0.02 \% \mathrm{CP}$ being stirred at $400 \mathrm{rpm}$ by a laboratory magnetic stirrer at room temperature for $8 \mathrm{~h}$ to completely evaporate the organic solvent. Then, TAT-RVC/NCs were harvested by ultracentrifugation $\left(15,000 \mathrm{rpm}, 4^{\circ} \mathrm{C}, 15 \mathrm{~min}\right)$ and resuspended in Milli-Q water. Then, the supernatant was collected and washed using Milli-Q water twice, resuspended in phosphate-buffered saline (PBS; $\mathrm{pH} 7.4$ ), filtered through a membrane (pore size of $0.45 \mu \mathrm{m}$ ), and lyophilized using $0.5 \%(\mathrm{w} / \mathrm{v})$ mannitol as a cryoprotectant.

Non-TAT-decorated RVC-loaded NCs (RVC/NCs) were prepared by the same method using PLGA-PEG instead of TAT-PEG-PLGA. Blank NCs were prepared by the same method without adding RVC.

\section{Drug encapsulation efficiency (EE) and drug-loading (DL) capacity}

Drug EE and DL capacity were evaluated by quantifying the amount of entrapped RVC in the NCs. ${ }^{26}$ To separate

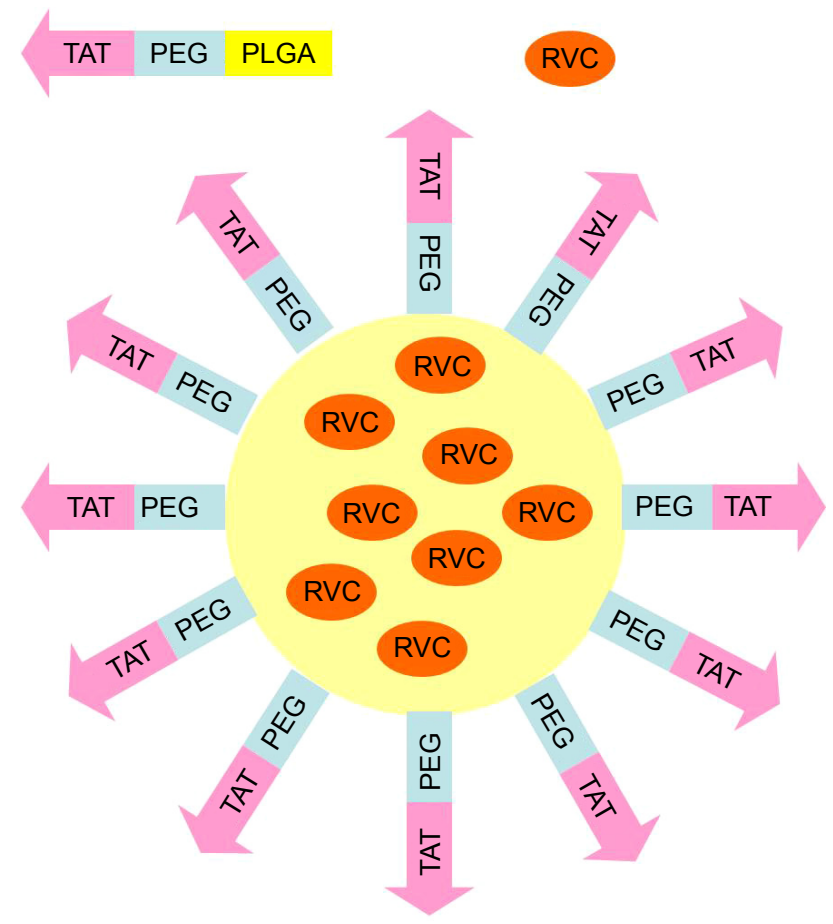

TAT-RVC/NCs

Figure I Structural schematic diagram of TAT-RVC/NCs.

Abbreviations: NCs, nanocarriers; PEG, polyethylene glycol; PLGA, poly(lactideco-glycolide); RVC, ropivacaine; TAT, transcriptional transactivator peptide; TATRVC/NCs, TAT-decorated RVC-loaded NCs.
RVC-loaded NCs from free RVC, Sephadex G-50 mini column centrifugation technique (size exclusion chromatography, SEC) was utilized. SEC is a chromatographic method in which molecules in solution are separated by their size, and in some cases the MW. It is generally applied to large molecules or macromolecular complexes such as proteins and polymers. Typically, when an aqueous solution is used to transport the sample through the column, the technique is known as gel-filtration chromatography, versus the name gel permeation chromatography, which is used when an organic solvent is used as a mobile phase. Briefly, $0.5 \mathrm{~mL}$ of TAT-RVC/NCs or RVC/NCs was placed onto a Sephadex G-50 mini column. The elution was conducted by adding $0.5 \mathrm{~mL}$ of distilled water and then centrifuging for $1 \mathrm{~min}$ at $500 \mathrm{rpm}$. Finally, the eluate was collected and mixed with methanol to break down the structure of NCs. The amount of entrapped RVC was detected by HPLC. The EE and DL of RVC-loaded NCs were calculated as follows:

$$
\begin{aligned}
& \mathrm{DL}(\%)=\frac{\text { Amount of RVC loaded in NCs }}{\text { All materials in the system }} \times 100 \\
& \mathrm{EE}(\%)=\frac{\text { Amount of RVC loaded in NCs }}{\text { Amount of RVC in the system }} \times 100
\end{aligned}
$$

\section{Mean diameter and zeta potential}

The measurements of mean diameter, polydispersity indices (PDIs), and zeta potential were performed by dynamic light scattering (photon correlation spectroscopy, PCS) using a ZetaSizer Nano ZS90 analyzer (Malvern Instruments, Malvern, UK). ${ }^{27}$

\section{Stability of TAT-RVC/NCs}

Stability of TAT-RVC/NCs, RVC/NCs, and NCs was evaluated by measuring mean diameter, PDI, zeta potential, and EE for 90 days. The suspensions were stored in amber flasks at ambient temperature. ${ }^{28}$ The results were calculated, and the analyses were performed at the predetermined time intervals $(0,15,30,60$, and 90 days $)$.

\section{In vitro drug release}

In vitro release behaviors of RVC from TAT-RVC/NCs, $\mathrm{RVC} / \mathrm{NCs}$, and RVC hydrochloride injection (RVC/IJ) in $\mathrm{PBS}$ at $37^{\circ} \mathrm{C}$ were evaluated for $48 \mathrm{~h}$ using a dialysis method. ${ }^{20}$ Briefly, $1 \mathrm{~mL}$ of solution was placed into a cellulose membrane dialysis tube ( $\mathrm{MWCO}=8,000-12,000$ ), suspended in $20 \mathrm{~mL}$ of PBS ( $\mathrm{pH} 7.4$ ), and gently shaken for $48 \mathrm{~h}$ at $37^{\circ} \mathrm{C}$ in a water bath at $100 \mathrm{rpm}$. At predetermined 
time points, $1 \mathrm{~mL}$ of releasing medium was withdrawn and refilled with the same amount of the fresh medium, and the released RVC was detected by HPLC.

\section{In vitro skin permeation study}

In vitro skin penetration experiments of TAT-RVC/NCs, RVC/NCs, and RVC/IJ were performed on Franz diffusion cells as follows: ${ }^{29,30} \mathrm{SD}$ rats were sacrificed, and the fur on the abdominal area of the rats was carefully removed. The skin from the abdominal surface was excised, and the adherent fat and subcutaneous tissue was removed. The receptor compartment was filled with $10 \mathrm{mM}$ phosphate buffer ( $\mathrm{pH} 7.4$ ), and the full thickness of skin was mounted on the diffusion cell with stratum corneum facing up and dermis being in contact with the receptor medium. The diffusion cells with the skin were maintained at $37^{\circ} \mathrm{C} \pm 0.5^{\circ} \mathrm{C}$ under non-occlusive conditions. The skin samples were mounted on Franz diffusion cell with a surface area of $0.66 \mathrm{~cm}^{2}$ and a receptor volume of $5 \mathrm{~mL}$. TAT-RVC/NCs, RVC/NCs, and RVC/IJ (containing $10 \mathrm{mg}$ of RVC) were applied on the donor compartment side of the skin, separately. Then, $0.5 \mathrm{~mL}$ of receptor medium was withdrawn at time intervals of $0,0.5,1,2,4,8,12,16,24$, and $48 \mathrm{~h}$ and was replaced by the same volume of fresh buffer to maintain the sink condition. The amount of RVC in the receptor fluid samples was assayed by HPLC. The flux of drug from each formulation was calculated. ${ }^{31}$ The cumulative drug permeation $\left(Q_{t}\right)$ was calculated from the following equation:

$$
Q_{t}=V_{r} C_{t}+\sum_{i=0}^{t-1} V_{s} C_{i}
$$

where $V_{r}$ and $V_{s}$ are the volumes of the receptor fluid; $C_{t}$ is the drug concentration of the receptor fluid; and $C_{i}$ is the drug concentration of the sample of the " $I$ " value. Data were reported in the cumulative drug permeation per unit of skin surface area, $Q_{t} / S\left(S=0.66 \mathrm{~cm}^{2}\right)$. The steady-state fluxes $\left(J_{S S}\right)$ were calculated by linear regression interpolation of the experimental data at steady state $(\Delta T)$ as shown in the following equation:

$$
J_{S S}=Q_{t} /(\Delta T \times S)
$$

\section{In vivo pharmacokinetics evaluation}

Important pharmacokinetics profiles of TAT-RVC/NCs, $\mathrm{RVC} / \mathrm{NCs}$, and RVC/IJ groups were evaluated. ${ }^{32}$ Rats were randomly divided into three groups ( $\mathrm{n}=6$ each) as follows: 1) TAT-RVC/NCs group; 2) RVC/NCs group; and 3) RVC/IJ group. The fur on the abdominal area was carefully removed. The next day, TAT-RVC/NCs and RVC/NCs were applied onto the skin, and RVC/IJ was applied by subcutaneous injection (1 mg RVC per rat). After 5, 10, 15, 20, 30, 45, 60, 75, and $90 \mathrm{~min}$, the skin tissue was collected and homogenized with 10 times the volume of PBS. The obtained homogenate was centrifuged at 10,000 rpm for $10 \mathrm{~min}$, and the supernate was collected. The amount of RVC in the supernate was detected by HPLC as mentioned earlier.

\section{In vivo anesthetic effect evaluation}

Tail-flick (TF) test was applied for evaluating the in vivo anesthetic effect of TAT-RVC/NCs, RVC/NCs, and $\mathrm{RVC} / \mathrm{IJ} .{ }^{33}$ Rats were randomly divided into four groups ( $\mathrm{n}=6$ each) as follows: 1) TAT-RVC/NCs group; 2) RVC/ NCs group; 3) RVC/IJ group; 4) 0.9\% saline group. A noxious heat stimulus was applied via a focused, radiant heat light source to the dorsal surface of the tail. TF test started 5 min after the local application of TAT-RVC/NCs, RVC/ $\mathrm{NCs}, \mathrm{RVC} / \mathrm{IJ}$, or $0.9 \%$ saline. TF latency was converted to represent the maximum possible effect (MPE) according to the following formula:

$$
\operatorname{MPE}(\%)=\frac{[(\text { Test latency })-(\text { Baseline latency })]}{[(\text { Cutoff time })-(\text { Baseline latency })]} \times 100 \text {. }
$$

The baseline latency was calculated as the mean of three different measurements taken at 15 min intervals. Baseline latencies were typically ranged from 2.5 to $3.0 \mathrm{~s}$. A maximum cutoff time of $10 \mathrm{~s}$ was set to avoid tissue damage in analgesic animals.

\section{Statistical analysis}

All data are expressed as mean \pm standard deviation. Statistical analyses were performed using Student's $t$-test. Differences were considered significant when the $P$-value was $<0.05$.

\section{Results}

\section{Preparation and characterization of TAT-RVC/NCs}

TAT-RVC/NCs were characterized by EE, DL, mean diameter, and zeta potential (Table 1). The EE was $81.7 \% \pm 3.1 \%$ and $82.4 \% \pm 2.8 \%$ and the DL was $8.2 \% \pm 0.8 \%$ and $9.3 \% \pm 0.9 \%$ for the TAT-RVC/NCs and RVC/NCs, respectively.

Mean diameter of TAT-RVC/NCs, RVC/NCs, and NCs was around $130 \mathrm{~nm}$, with narrow PDIs (below 0.2). 
Table I Characterization of TAT-RVC/NCs, RVC/NCs, and NCs

\begin{tabular}{llllll}
\hline Formulation & EE & DL & Mean diameter & PDI & Zeta potential \\
\hline TAT-RVC/NCs & $81.7 \pm 3.1$ & $8.2 \pm 0.8$ & $133.2 \pm 4.6$ & $0.193 \pm 0.021$ & $-19.1 \pm 1.3$ \\
RVC/NCs & $82.4 \pm 2.8$ & $9.3 \pm 0.9$ & $134.1 \pm 3.7$ & $0.127 \pm 0.018$ & $-27.8 \pm 2.4$ \\
NCs & N/A & N/A & $128.9 \pm 3.1$ & $0.091 \pm 0.009$ & $-26.3 \pm 1.7$ \\
\hline
\end{tabular}

Note: Data represent mean \pm SD $(n=3)$.

Abbreviations: DL, drug loading; EE, encapsulation efficiency; NCs, nanocarriers; PDI, polydispersity index; RVC, ropivacaine; SD, standard deviation; TAT, transcriptional transactivator peptide; RVC/NCs, RVC-loaded NCs; TAT-RVC/NCs, TAT-decorated RVC-loaded NCs; N/A, not available.

The average diameter of TAT-RVC/NCs obtained in this study was $130 \mathrm{~nm}$. The zeta potential of TAT-RVC/NCs was $-19.1 \mathrm{mV}$.

\section{Stability of the TAT-RVC/NCs}

The stabilities of TAT-RVC/NCs, RVC/NCs, and NCs were evaluated over a period of 90 days of storage in amber flasks at ambient temperature. For both kinds of NCs, the average particle diameter remained almost constant throughout the period (Figure 2A). There were no significant changes with time of the PDIs of all the formulations tested (Figure 2B). For both formulations, the zeta potential was negative and showed no major changes during the 90 days of storage (Figure 2C). The RVC EE of TAT-RVC/NCs and RVC/NCs
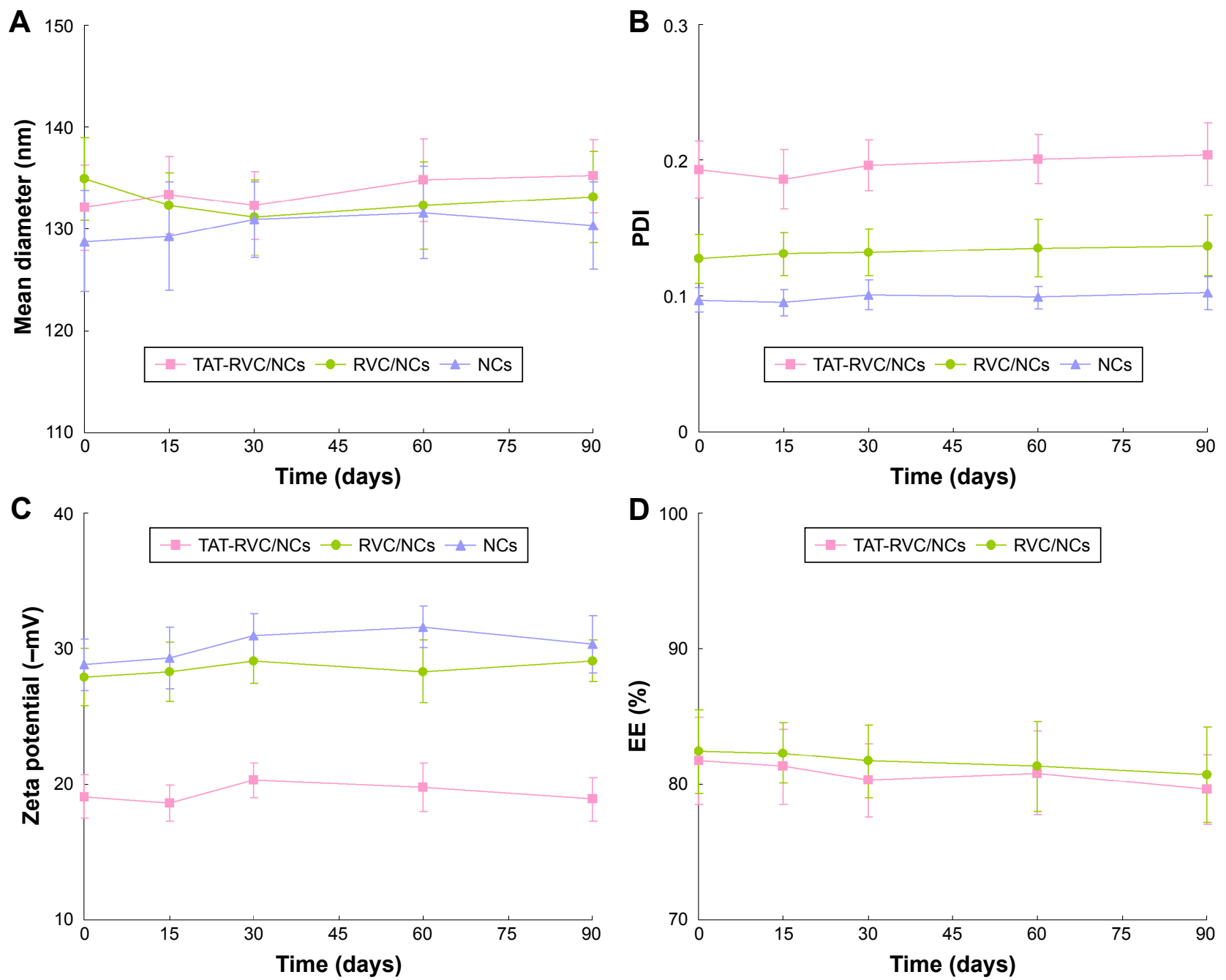

Figure 2 Stabilities of TAT-RVC/NCs, RVC/NCs, and NCs in terms of mean particle diameter (A), PDI (B), zeta potential (C), and EE (D). Note: Data represent mean \pm SD $(n=3)$.

Abbreviations: EE, encapsulation efficiency; NCs, nanocarriers; PDI, polydispersity index; RVC, ropivacaine; SD, standard deviation; TAT, transcriptional transactivator peptide; RVC/NCs, RVC-loaded NCs; TAT-RVC/NCs, TAT-decorated RVC-loaded NCs. 
also remained stable at all the time points tested in the research (Figure 2D).

\section{In vitro drug release}

In vitro drug release profiles of TAT-RVC/NCs, RVC/NCs, and RVC/IJ are shown in Figure 3. Significant differences in the release patterns were observed between drug injection and drug-loaded NCs $(P<0.05)$. The drug-loaded NCs led to a slower release rate of RVC compared with injection. It was clear that encapsulation into polymeric NCs exhibited an obvious sustained release.

\section{In vitro skin permeation}

In vitro permeation behaviors of TAT-RVC/NCs, RVC/NCs, and RVC/IJ were calculated (Figure 4A), and the $J_{S S}$ were summarized (Figure 4B). The $J_{S S}$ of TAT-RVC/NCs, RVC/ $\mathrm{NCs}$, and RVC/IJ were 19.7 $\pm 1.1,14.2 \pm 1.2$, and 8.6 \pm 0.8 , respectively. The permeation of RVC loaded in NCs was much more sufficient than RVC injection $(P<0.05)$. The highest skin permeation was provided by TAT-RVC/NCs. The $J_{S S}$ of TAT-RVC/NCs was significantly higher than that of RVC/NCs $(P<0.05)$.

\section{In vivo pharmacokinetics evaluation}

Pharmacokinetic profiles of TAT-RVC/NCs, RVC/NCs, and $\mathrm{RVC} / \mathrm{IJ}$ after administration to rat skin are shown in Figure 5. RVC/IJ showed the highest tissue level of itself $(98 \mu \mathrm{g} / \mathrm{g}$ weight) at $10 \mathrm{~min}$ after injected to the skin. TAT-RVC/NCs

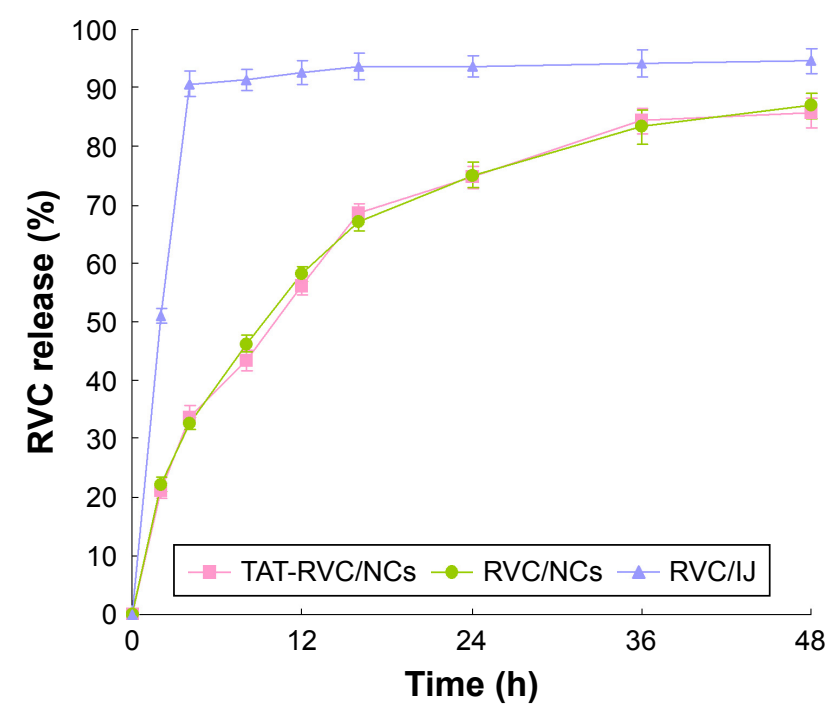

Figure 3 In vitro drug release profiles of TAT-RVC/NCs, RVC/NCs, and RVC/IJ. Note: Data represent mean $\pm S D(n=3)$.

Abbreviations: NCs, nanocarriers; RVC, ropivacaine; SD, standard deviation; TAT, transcriptional transactivator peptide; RVC/NCs, RVC-loaded NCs; TATRVC/NCs, TAT-decorated RVC-loaded NCs; RVC/IJ, RVC hydrochloride injection. and RVC/NCs showed sustained-release behavior and the highest skin tissue RVC levels were reached at $4 \mathrm{~h}$ after application. TAT-RVC/NCs showed maximum tissue RVC level, higher than that of RVC/NCs and RVC/IJ.

\section{In vivo anesthetic effect}

In vivo anesthetic effect of TAT-RVC/NCs, RVC/NCs, and RVC/IJ was reviewed by TF test. TAT-RVC/NCs, RVC/ $\mathrm{NCs}$, and RVC/IJ increased the TF latency dramatically (Figure 6). There were no significant differences in baseline TF latency among all the groups tested. The time MPE in the saline group showed no obvious change during the experiment. RVC-loaded NCs showed fast and effective, and also long-lasting effect; on the contrary, the RVC/IJ showed a fast but short-lasting anesthetic effect. TAT-RVC/NCs induced a remarkable anesthetic effect, persisting until 45 min after the administration.

\section{Discussion}

Polymeric nanoparticles for the delivery of LAs via the skin bring a lot of advantages such as delivering the local drug continuously and reducing systemic adverse reactions. ${ }^{34}$ TAT can successfully enhance the transepidermal absorption of therapeutic molecules for the local therapy. In this study, TAT-decorated RVC was encapsulated and NC system was prepared and used to enhance the local anesthesia of RVC.

Table 1 summarizes the EE, DL, mean diameter, and zeta potential of NCs. Quantification of the amount of drug encapsulated in the systems could consider the RVC EE and DL differences between the TAT-decorated TAT-RVC/ NCs and the non-decorated RVC/NCs. ${ }^{27}$ The EE and DL for the TAT-RVC/NCs and RVC/NCs had no obvious difference $(P>0.05)$, suggesting that the decoration of TAT did not affect the drug encapsulation. The NCs prepared were relatively uniform and homogenous, due to narrow PDIs (below 0.2) were found in TAT-RVC/NCs, RVC/NCs, and NCs. Size was critical to topical drug delivery systems, since it was reported that vesicles smaller than $300 \mathrm{~nm}$ were able to deliver the drug into deeper layers of the skin. ${ }^{35}$ The average diameter of TAT-RVC/NCs obtained in this study was $130 \mathrm{~nm}$; this may be the evidence that the formulation had potential for skin delivery of drugs. The stability of the NCs can also be influenced by the charge of the system. Since aggregation could be avoided by electrostatic repulsion between vesicles, higher zeta potential means better long-term stability. ${ }^{26}$ The zeta potential of TAT-RVC/NCs was $-19.1 \mathrm{mV}$, which may forecast a fine stability for the formulation. 

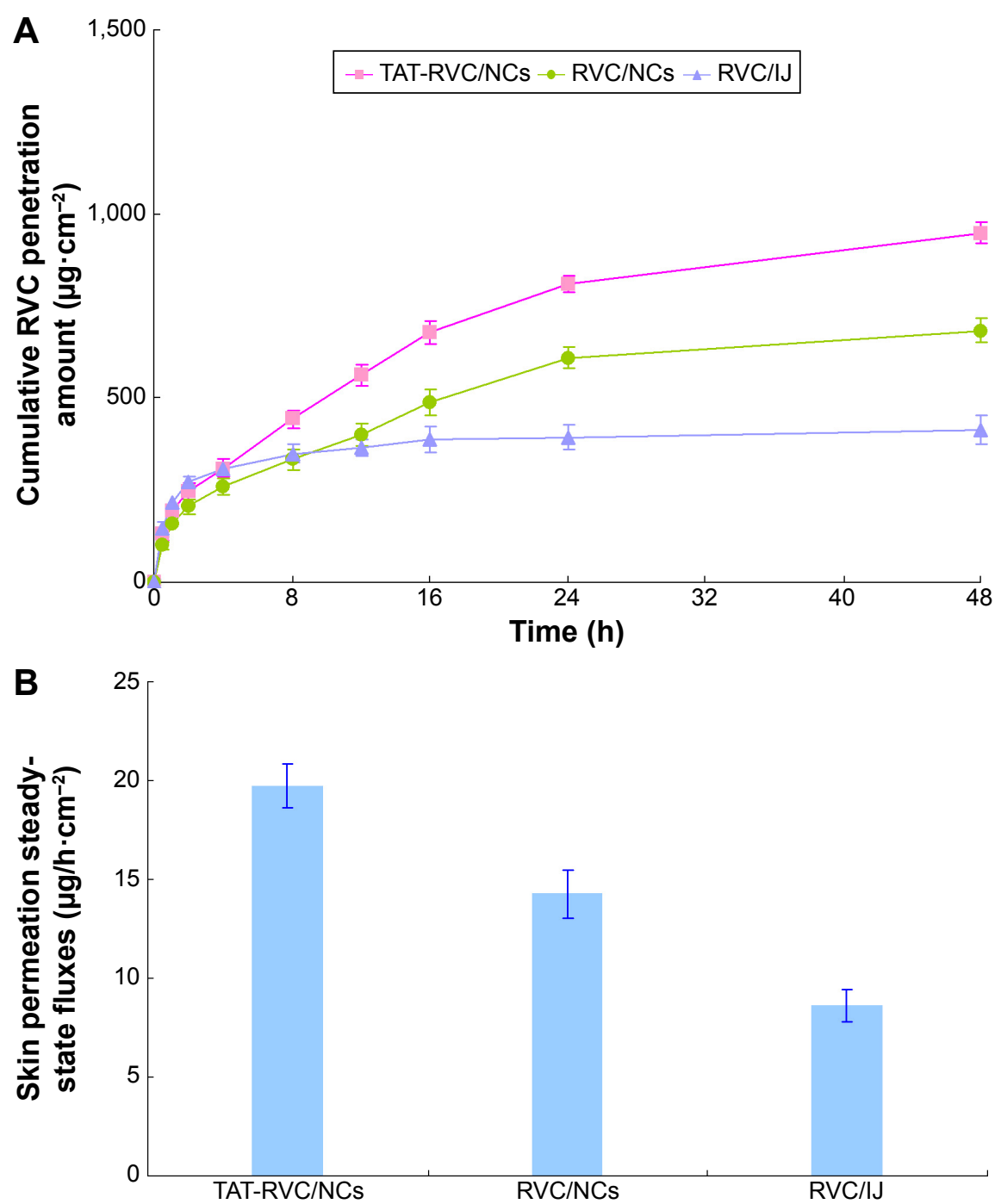

Figure 4 In vitro permeation behaviors (A) and the steady-state fluxes (B) of TAT-RVC/NCs, RVC/NCs, and RVC/IJ.

Note: Data represent mean $\pm S D(n=3)$.

Abbreviations: NCs, nanocarriers; RVC, ropivacaine; SD, standard deviation; TAT, transcriptional transactivator peptide; RVC/NCs, RVC-loaded NCs; TAT-RVC/NCs, TAT-decorated RVC-loaded NCs; RVC/IJ, RVC hydrochloride injection.

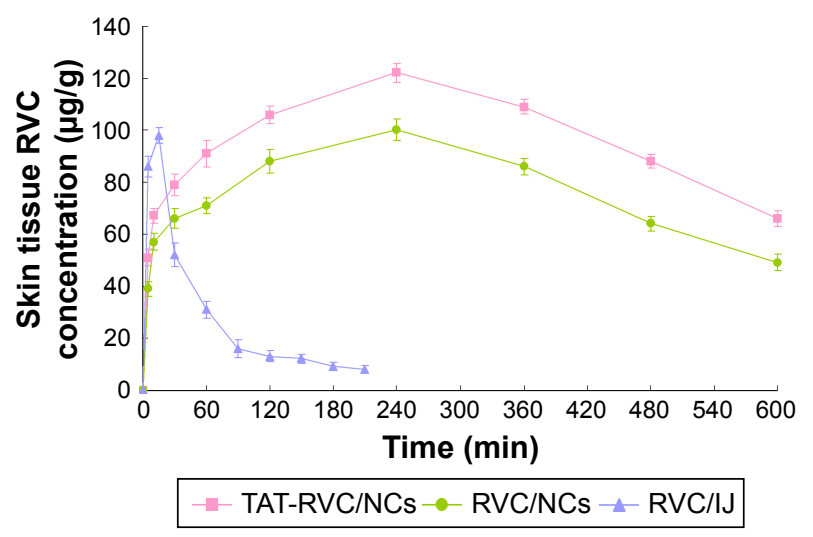

Figure 5 Pharmacokinetic profiles of RVC after TAT-RVC/NCs, RVC/NCs, and RVC/IJ were administrated to rat skin.

Note: Data represent mean \pm SD $(n=6)$.

Abbreviations: NCs, nanocarriers; RVC, ropivacaine; SD, standard deviation; TAT, transcriptional transactivator peptide; RVC/NCs, RVC-loaded NCs; TATRVC/NCs, TAT-decorated RVC-loaded NCs; RVC/IJ, RVC hydrochloride injection.
The stabilities of TAT-RVC/NCs, RVC/NCs, and NCs were evaluated over a period of 90 days in terms of the mean particle diameter, PDI, zeta potential, and EE. For both kinds of NCs, no aggregate was found for the average particle diameter remained almost constant throughout the period. PDIs less than 0.2 are normally used to indicate the existence of a narrow particle size range. Large fluctuations with time could indicate aggregate formation, hence increasing the nanoparticle size range. ${ }^{36}$ There were no significant changes with time of the PDIs of all the formulations tested, showing the stability of the NCs. The value of the zeta potential also provides an indication of the stability of NCs in suspension. ${ }^{37}$ For both formulations, the zeta potential was negative and showed no major changes during the 90 days of storage. The RVC EE of TAT-RVC/NCs and RVC/NCs also remained 


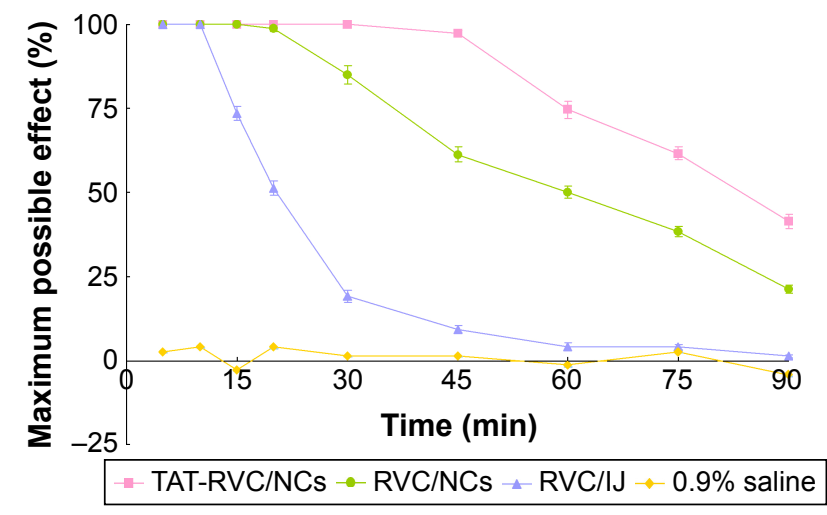

Figure 6 In vivo anesthetic effect of TAT-RVC/NCs, RVC/NCs, and RVC/IJ. Note: Data represent mean $\pm S D(n=6)$.

Abbreviations: NCs, nanocarriers; RVC, ropivacaine; SD, standard deviation; TAT, transcriptional transactivator peptide; RVC/NCs, RVC-loaded NCs; TATRVC/NCs, TAT-decorated RVC-loaded NCs; RVC/IJ, RVC hydrochloride injection.

stable at all the time points tested in the research, indicating that the NCs were stable within 3 months.

In vitro RVC release profiles of TAT-RVC/NCs, RVC/ $\mathrm{NCs}$, and RVC/IJ illustrated that the RVC released from NCs in sustained manners. The sustained-release behavior is an important prerequisite for successful drug delivery. ${ }^{38}$ This phenomenon could be explained by the entrapment of the drugs by the polymer which can slow down the release of the drugs. Complete releases of drug in TAT-RVC/NCs and RVC/NCs were achieved at $48 \mathrm{~h}$. This could contribute to the use of PLGA-PEG which incorporated RVC and let it release in a more sustained rate.

In vitro permeation study results showed that the permeation of RVC in TAT-RVC/NCs was much more sufficient than RVC/NCs and RVC/IJ $(P<0.05)$. The results illustrated that NCs have better skin permeation capacity, which may due to the nanometric size, and the biocompatibility for long-term skin administration. ${ }^{39,40}$ TAT, one of the most frequently used cell penetrating peptides, has been successfully used to facilitate the delivery of pharmaceutical NCs to the cell interior both in vitro and in vivo. ${ }^{41}$ In this study, after TAT decoration was accomplished, its penetration rate and flux were significantly higher than non-decorated RVC/NCs $(P<0.05)$. These results indicated that TAT has the potential to help the NCs to cross the skin layers more efficiently than other systems.

In vivo pharmacokinetic profiles of TAT-RVC/NCs, $\mathrm{RVC} / \mathrm{NCs}$, and RVC/IJ were compared. TAT-RVC/NCs and RVC/NCs showed sustained-release behavior, and TAT-RVC/NCs showed maximum tissue RVC level after administration to the skin. This could reveal the improved bioavailability of TAT-RVC/NCs, which are corresponding to the increased efficacy.
In vivo TF test is most frequently used to measure pain levels and assess the effects of anesthesia. ${ }^{42}$ There were no significant differences in baseline TF latency among all the groups tested. The time MPE in the saline group showed no obvious change during the experiment. RVC-loaded NCs showed fast and effective, and also long-lasting effect; on the contrary, the RVC/IJ showed a fast but short-lasting anesthetic effect. The results indicated that the anesthetic activity of TAT-RVC/NCs revealed a more interesting anesthetic effect in the first few minutes and also showed sustained anesthetic activity compared with the RVC/IJ. The sustained and impressive anesthetic effect of the TAT-RVC/ NCs could bring about better LA therapy effects than other formula tested. Thus, outstanding permeation efficiency of the TAT-decorated NCs could deliver RVC deeper in the skin, thereby enhancing the in vivo anesthetic effect of the system.

\section{Conclusion}

It can be concluded that the skin permeation efficiency of RVC from TAT-decorated NCs was increased. This could be explained by the fact that nano-sized NCs have the ability to permeate through the skin surface. In vitro and in vivo studies showed that TAT-RVC/NCs more effectively promoted skin permeation capacity and anesthetic effect. Therefore, TAT-decorated NCs can be used as an effective transdermal delivery system for local anesthesia.

\section{Disclosure}

The authors report no conflicts of interest in this work.

\section{References}

1. Akerman B, Hellberg IB, Trossvik C. Primary evaluation of the local anaesthetic properties of the amino amide agent ropivacaine (LEA 103). Acta Anaesthesiol Scand. 1988;32(7):571-578.

2. McClure JH. Ropivacaine. Br J Anaesth. 1996;76(2):300-307. Review.

3. Halldin MM, Bredberg E, Angelin B, et al. Metabolism and excretion of ropivacaine in humans. Drug Metab Dispos. 1996;24(9):962-968.

4. Zink W, Graf BM. The toxicity of local anesthetics: the place of ropivacaine and levobupivacaine. Curr Opin Anaesthesiol. 2008;21(5): 645-650.

5. Ratajczak-Enselme M, Estebe JP, Dollo G, et al. Epidural, intrathecal and plasma pharmacokinetic study of epidural ropivacaine in PLGAmicrospheres in sheep model. Eur J Pharm Biopharm. 2009;72(1):54-61.

6. Li TF, Fan H, Wang YX. Epidural sustained release ropivacaine prolongs anti-allodynia and anti-hyperalgesia in developing and established neuropathic pain. PLoS One. 2015;10(1):e0117321.

7. Scott DB, Lee A, Fagan D, Bowler GM, Bloomfield P, Lundh R. Acute toxicity of ropivacaine compared with that of bupivacaine. Anesth Analg. 1989;69(5):563-569

8. Tam KW, Chen SY, Huang TW, et al. Effect of wound infiltration with ropivacaine or bupivacaine analgesia in breast cancer surgery: a metaanalysis of randomized controlled trials. Int J Surg. 2015;22:79-85. 
9. Zhai Y, Zhao L, Wang Z, Zhai G. Retracted article: preparation and characterization of novel lipid nanocapsules of ropivacaine for transdermal delivery. Drug Deliv. 2016;23(2):619-628.

10. Leone S, Di Cianni S, Casati A, Fanelli G. Pharmacology, toxicology, and clinical use of new long acting local anesthetics, ropivacaine and levobupivacaine. Acta Biomed. 2008;79(2):92-105.

11. de Araújo DR, da Silva DC, Barbosa RM, et al. Strategies for delivering local anesthetics to the skin: focus on liposomes, solid lipid nanoparticles, hydrogels and patches. Expert Opin Drug Deliv. 2013;10(11):1551-1563.

12. Sharma G, Kamboj S, Thakur K, Negi P, Raza K, Katare OP. Delivery of thermoresponsive-tailored mixed micellar nanogel of lidocaine and prilocaine with improved dermatokinetic profile and therapeutic efficacy in topical anaesthesia. AAPS PharmSciTech. 2017;18(3):790-802.

13. Hassan AO, Elshafeey AH. Nanosized particulate systems for dermal and transdermal delivery. J Biomed Nanotechnol. 2010;6(6):621-633.

14. Luengo J, Weiss B, Schneider M, et al. Influence of nanoencapsulation on human skin transport of flufenamic acid. Skin Pharmacol Physiol. 2006;19(4):190-197.

15. Gou M, Wu L, Yin Q, et al. Transdermal anaesthesia with lidocaine nano-formulation pretreated with low-frequency ultrasound in rats model. J Nanosci Nanotechnol. 2009;9(11):6360-6365.

16. Shim J, Seok Kang H, Park WS, Han SH, Kim J, Chang IS. Transdermal delivery of mixnoxidil with block copolymer nanoparticles. J Control Release. 2004;97(3):477-484.

17. Lohcharoenkal W, Manosaroi A, Götz F, Werner RG, Manosroi W, Manosaroi J. Potent enhancement of GFP uptake into HT-29 cells and rat skin permeation by coincubation with tat peptide. J Pharm Sci. 2011; 100(11):4766-4773.

18. Manosroi J, Lohcharoenkal W, Götz F, Werner RG, Manosroi W, Manosroi A. Transdermal absorption and stability enhancement of salmon calcitonin by Tat peptide. Drug Dev Ind Pharm. 2013;39(4): 520-525.

19. Chen X, Liu S, Rao P, Bradshaw J, Weller R. Topical application of superoxide dismutase mediated by HIV-TAT peptide attenuates UVB-induced damages in human skin. Eur J Pharm Biopharm. 2016;107:286-294.

20. Wang Y, Su W, Li Q, et al. Preparation and evaluation of lidocaine hydrochloride-loaded TAT-conjugated polymeric liposomes for transdermal delivery. Int J Pharm. 2013;441(1-2):748-756.

21. Wang Y, Wang S, Shi P. Transcriptional transactivator peptide modified lidocaine-loaded nanoparticulate drug delivery system for topical anesthetic therapy. Drug Deliv. 2016;23(9):3193-3199.

22. Koren E, Apte A, Sawant RR, Grunwald J, Torchilin VP. Cell-penetrating TAT peptide in drug delivery systems: proteolytic stability requirements. Drug Deliv. 2011;18(5):377-384.

23. Zhu Y, Cheng L, Cheng L, et al. Folate and TAT peptide co-modified liposomes exhibit receptor-dependent highly efficient intracellular transport of payload in vitro and in vivo. Pharm Res. 2014;31(12): 3289-3303.

24. Zou W, Liu C, Chen Z, Zhang N. Studies on bioadhesive PLGA nanoparticles: a promising gene delivery system for efficient gene therapy to lung cancer. Int J Pharm. 2009;370(1-2):187-195.

25. Zou W, Cao G, Xi Y, Zhang N. New approach for local delivery of rapamycin by bioadhesive PLGA-carbopol nanoparticles. Drug Deliv. 2009;16(1):15-23.
26. Zhai Y, Xu R, Wang Y, Liu J, Wang Z, Zhai G. Ethosomes for skin delivery of ropivacaine: preparation, characterization and ex vivo penetration properties. J Liposome Res. 2015;25(4):316-324.

27. Silva de Melo NF, Campos EV, Gonçalves CM, et al. Development of hydrophilic nanocarriers for the charged form of the local anesthetic articaine. Colloids Surf B Biointerfaces. 2014;121:66-73.

28. de Melo NF, Grillo R, Guilherme VA, et al. Poly(lactide-co-glycolide) nanocapsules containing benzocaine: influence of the composition of the oily nucleus on physico-chemical properties and anesthetic activity. Pharm Res. 2011;28(8):1984-1994.

29. Vidlářová L, Hanuš J, Veselý M, Ulbrich P, Štěpánek F, Zbytovská J. Effect of lipid nanoparticle formulations on skin delivery of a lipophilic substance. Eur J Pharm Biopharm. 2016;108:289-296.

30. Wang J, Zhang L, Chi H, Wang S. An alternative choice of lidocaineloaded liposomes: lidocaine-loaded lipid-polymer hybrid nanoparticles for local anesthetic therapy. Drug Deliv. 2016;23(4):1254-1260.

31. Junyaprasert VB, Boonme P, Wurster DE, Rades T. Aerosol OT microemulsions as carriers for transdermal delivery of hydrophobic and hydrophilic local anesthetics. Drug Deliv. 2008;15(5):323-330.

32. Ito Y, Ohta J, Imada K, et al. Dissolving microneedles to obtain rapid local anesthetic effect of lidocaine at skin tissue. J Drug Target. 2013; 21(8):770-775.

33. Ouchi K, Sekine J, Koga Y, Nakao S, Sugiyama K. Establishment of an animal model of sedation using epidural anesthesia that uses the tail-flick test for evaluating local anesthetic effects in rats. Exp Anim. 2013;62(2):137-144.

34. Peptu C, Rotaru R, Ignat L, et al. Nanotechnology approaches for pain therapy through transdermal drug delivery. Curr Pharm Des. 2015;21(42):6125-6139.

35. Verma DD, Verma S, Blume G, Fahr A. Particle size of liposomes influences dermal delivery of substances into skin. Int J Pharm. 2003; 258(1-2):141-151.

36. Mohanraj VJ, Chen Y. Nanoparticles - a review. Trop JPharm Res. 2006; 5:561-573.

37. Song Z, Lu Y, Zhang X, Wang H, Han J, Dong C. Novel curcuminloaded human serum albumin nanoparticles surface functionalized with folate: characterization and in vitro/vivo evaluation. Drug Des Devel Ther. 2016;10:2643-2649.

38. Feng C, Li X, Dong C, Zhang X, Zhang X, Gao Y. RGD-modified liposomes enhance efficiency of aclacinomycin A delivery: evaluation of their effect in lung cancer. Drug Des Devel Ther. 2015;9:4613-4620.

39. Ni Q, Chen W, Tong L, Cao J, Ji C. Preparation of novel biodegradable ropivacaine microspheres and evaluation of their efficacy in sciatic nerve block in mice. Drug Des Devel Ther. 2016;10:2499-2506.

40. Vitorino C, Almeida J, Gonçalves LM, Almeida AJ, Sousa JJ, Pais AA. Co-encapsulating nanostructured lipid carriers for transdermal application: from experimental design to the molecular detail. J Control Release. 2013;167(3):301-314.

41. Torchilin VP. Cell penetrating peptide-modified pharmaceutical nanocarriers for intracellular drug and gene delivery. Biopolymers. 2008;90(5):604-610.

42. Kwon SS, Kim SY, Kong BJ, et al. Cell penetrating peptide conjugated liposomes as transdermal delivery system of Polygonum aviculare L. extract. Int J Pharm. 2015;483(1-2):26-37.

\section{Publish your work in this journal}

Drug Design, Development and Therapy is an international, peerreviewed open-access journal that spans the spectrum of drug design and development through to clinical applications. Clinical outcomes, patient safety, and programs for the development and effective, safe, and sustained use of medicines are the features of the journal, which

\section{Dovepress}

has also been accepted for indexing on PubMed Central. The manuscript management system is completely online and includes a very quick and fair peer-review system, which is all easy to use. Visit http://www.dovepress.com/testimonials.php to read real quotes from published authors. 